Supporting Information

\title{
The Role of Fluorinated Aryl Ether Moiety in Polyimide-co-etherimide on
} Gas Transport Properties

\author{
Susilo Japip ${ }^{1}$, Guang Rong Lee ${ }^{2}$, Tai-Shung Chung ${ }^{1 *}$ \\ ${ }^{1}$ Department of Chemical and Biomolecular Engineering, \\ National University of Singapore, 117585, Singapore \\ ${ }^{2}$ Engineering Science Programme, National University of Singapore, 117575, Singapore \\ *Corresponding author \\ Email: chencts@nus.edu.sg \\ Fax : (65)-67791936
}




\section{S1. EXPERIMENTAL SECTION}

\section{S1.1. Synthesis of Fluorinated Polyimide-co-etherimides}

Each fluorinated polyimide-co-etherimide (6FDA-durene-4BDAF) was synthesized in-house via a conventional polycondensation method in anhydrous NMP. Prior to use, 6FDA and 4BDAF were purified via vacuum sublimation method, whilst durene was recrystallized in methanol and NMP was vacuum distilled at $65^{\circ} \mathrm{C}$. Typically, certain amounts of durene and 4BDAF were dissolved in a freshly distilled NMP with a total solid concentration of $20 \mathrm{wt} \%$ at room temperature in an inert atmosphere. The solution was stirred for half an hour prior to the addition of an equimolar amount of 6FDA (relative to the total concentration of diamine), after which it was continuously stirred for $24 \mathrm{~h}$ in an inert atmosphere. The intermediate polyamic acid was converted into polyimide via the chemical imidization method by adding acetic anhydride and TEA into the solution with a 4 to 1 molar ratio. The mixture was further left to stir for another $24 \mathrm{~h}$ followed by precipitation in a methanol solution. Fresh methanol was changed for at least three times before the solidified polyimide-co-etherimide was dried at $65^{\circ} \mathrm{C}$ in a Heraeus vacuum oven overnight.

\section{S1.2. Detailed Characterization Method and Condition}

The GPC system consisted of a Waters e2695 Alliance HPLC system and a Waters 2414 refractive index detector, which coupled two Agilent Polargel (IM) columns in series (7.8 x $300 \mathrm{~mm}$ ) together with a Polargel (IM) Guard column. HPLC grade DMF was selected as the mobile phase, whereas PMMA EasiVial tri-pack standards (Agilent) were used for calibration. The measurements were conducted at column and detector temperatures of $60^{\circ} \mathrm{C}$ and $50^{\circ} \mathrm{C}$, respectively, with a mobile phase flow rate of $1 \mathrm{~mL} \mathrm{~min}{ }^{-1}$ and each sample concentration of 5 $\mathrm{mg} \mathrm{mL}^{-1}$. 
The $d$-spacing of the fabricated membranes was analyzed by employing X-ray diffraction (XRD) on a Bruker D8 Advance X-ray diffractometer using $\mathrm{Cu}-\mathrm{K} \alpha$ as the $\mathrm{X}$-ray source with a wavelength of $1.54 \AA$. The average $d$-spacing can be calculated according to the Bragg's law as provided in equation (S1).

$n \lambda=2 d \sin \theta$

where $n$ is an integer, $\lambda$ represents the wavelength of the incoming X-ray source, $d$ refers to the inter-segmental distance between two adjacent polymer chains and $\theta$ signifies the diffraction angle of the diffracted X-ray.

The fractional free volume (FFV) variation of the fabricated membranes was elucidated employing positron annihilation lifetime spectroscopy (PALS) on a conventional fast-fast coincidence spectroscope. A radioactive source of ${ }^{22} \mathrm{Na}$ sandwiched between two Kapton ${ }^{\circledR}$ films was utilized as the source of positrons. A minimally $1 \mathrm{~mm}$ thick stack of samples was used to sandwich the ${ }^{22} \mathrm{Na}$ source. Each spectrum was collected with a total of five million counts. The PALS spectra were then resolved into four-lifetime components using a PATFIT88 program to obtain the mean positron lifetime. The four-lifetime components consist of the annihilation of para-Positronium ( $p$-Ps, $\tau_{1} \approx 0.125 \mathrm{~ns}$ ), free positron $\left(\tau_{2} \approx 0.4 \mathrm{~ns}\right)$, and localized ortho-Positronium (o-Ps, $\tau_{3}$ and $\tau_{4}>0.6 \mathrm{~ns}$ ) in the membrane free volume with their corresponding intensities ( $I_{1}, I_{2}, I_{3}$, and $I_{4}$, respectively). The free-volume radius, $R(\AA)$, of each membrane was calculated based on an established semi-empirical correlation equation of the $o$-Ps for spherical cavity as provided in equation (S2) below. ${ }^{1-3}$

$$
\tau_{i}^{-1}=2\left[1-\frac{R}{\Delta R}+\frac{1}{2 \pi} \sin \left(\frac{2 \pi R}{\Delta R}\right)\right]
$$


where $\tau_{i}=\tau_{3}$ or $\tau_{4}$ are the $o$-Ps lifetime (ns), and $\Delta R$ indicates an empirical parameter (1.66 $\AA$ ). The fractional free volume (FFV) can be determined by means of the Williams-LandelFerry equation according to the spherical cavity assumption as follows. ${ }^{3,4}$

$$
F F V=\sum_{i} 0.0018 I_{i}\left(\frac{4}{3} \pi R_{i}^{3}\right)
$$

where $I_{i}=I_{3}$ or $I_{4}$ denote the $o$-Ps intensities (\%) and $R_{i}=R_{3}$ or $R_{4}$ refer to the free-volume radius $(\AA)$.

\section{S2. RESULTS AND DISCUSSION}

\section{S2.1. Relationship of Logarithmic Permeability versus Volume Fraction of 6FDA-4BDAF}

Figure S1 displays the linear relationship of the logarithmic permeability versus the volume fraction of 6FDA-4BDAF moiety. The volume fraction of 6FDA-4BDAF in the copolymers can be calculated according to equation (S4) as follows.

Volume fraction $=\frac{V_{S 1} \omega_{1}}{V_{S 1} \omega_{1}+V_{S 2} \omega_{2}}$

Where $V_{S}$ denotes specific volume which was obtained by taking the reciprocal of density (in this study, we used the density obtained by helium pycnometry), $\omega$ refers to the weight fraction obtained from ${ }^{1} \mathrm{H}$ NMR and is depicted in Table 1, and subscripts 1 and 2 represent 6FDA4BDAF and 6FDA-durene moieties, respectively.

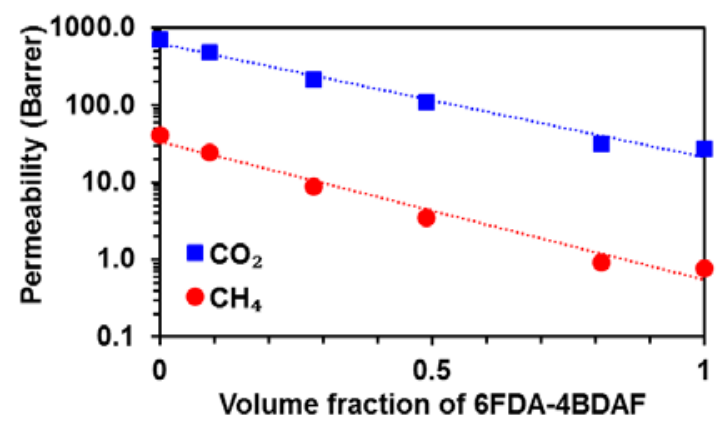

Figure S1. Effect of 6FDA-4BDAF volume fraction on $\mathrm{CO}_{2}$ and $\mathrm{CH}_{4}$ permeability of the fabricated membranes. 


\section{S2.2. Gas Transport Properties according to Free Volume Theory}

Figures S2 and S3 demonstrate the correlations of $\mathrm{CO}_{2}$ and $\mathrm{CH}_{4}$ diffusivity coefficients and $\mathrm{CO}_{2} / \mathrm{CH}_{4}$ diffusivity, respectively, versus the reciprocal of fractional free volume (FFV) of the membranes. These correlations are based on the free volume theory which has been widely utilized to describe the transport of small molecules in a variety of non-porous polymers as follows. ${ }^{5-8}$

$D_{i}=A_{i} \exp \left[-\frac{B_{i}}{F F V}\right]$

Where $D$ is the diffusivity coefficient, $A$ and $B$ are adjustable constants and subscript $i$ represents the penetrant molecule, which is $\mathrm{CO}_{2}$ and $\mathrm{CH}_{4}$ in this study.

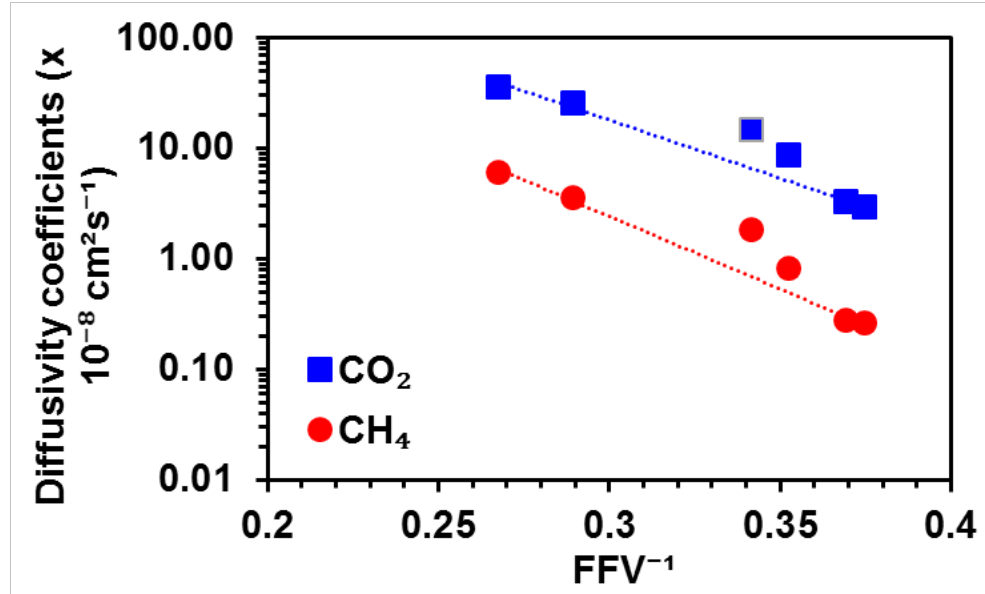

Figure S2. Correlation between $\mathrm{CO}_{2}$ and $\mathrm{CH}_{4}$ diffusivity coefficients vs. reciprocal of FFV.

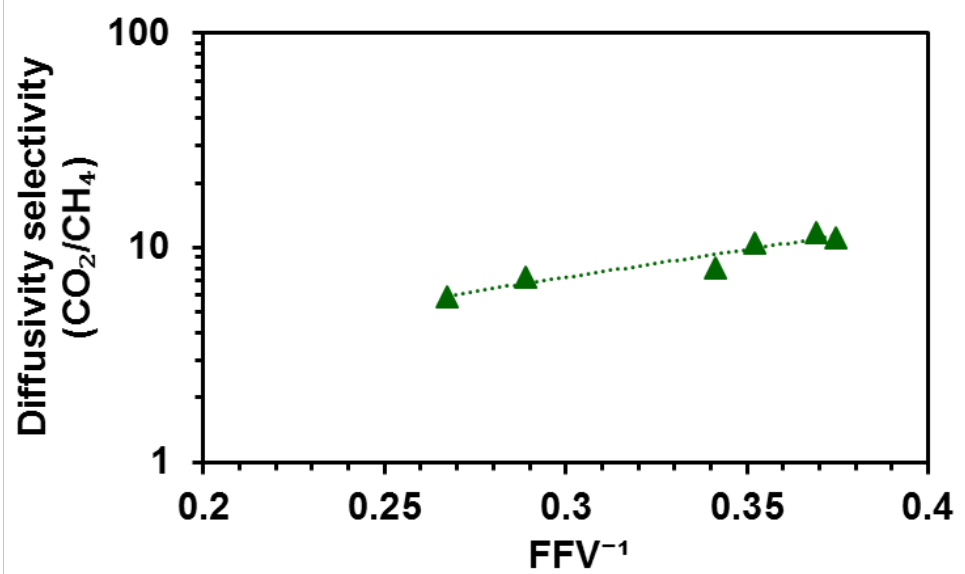

Figure S3. Correlation between $\mathrm{CO}_{2} / \mathrm{CH}_{4}$ diffusivity selectivity vs. reciprocal of FFV. 


\section{S2.3. Fitted Dual Mode Langmuir-Henry Parameters}

Table S1. Fitted dual mode Langmuir-Henry parameters for $\mathrm{CO}_{2}$ and $\mathrm{CH}_{4}$ adsorption isotherms.

\begin{tabular}{lcccccc}
\hline \multirow{2}{*}{\multicolumn{1}{c}{ Membrane }} & \multicolumn{3}{c}{$\mathbf{C O}_{\mathbf{2}}$} & \multicolumn{3}{c}{$\mathbf{C H}_{\mathbf{4}}$} \\
\cline { 2 - 7 } & $\mathbf{k}_{\mathbf{D}}$ & $\mathbf{C}_{\mathbf{H}}^{\prime}$ & $\mathbf{b}$ & $\mathbf{k}_{\mathbf{D}}$ & $\mathbf{C}_{\mathbf{H}}^{\prime}$ & $\mathbf{b}$ \\
\hline 6FDA-durene & 3.15 & 54.8 & 0.75 & 1.06 & 37.1 & 0.17 \\
6FDA-durene-4BDAF (9:1) & 3.12 & 50.0 & 0.78 & 0.70 & 47.3 & 0.12 \\
6FDA-durene-4BDAF (7:3) & 2.72 & 42.6 & 0.80 & 0.63 & 27.8 & 0.14 \\
6FDA-durene-4BDAF (5:5) & 2.48 & 35.1 & 0.73 & 0.69 & 25.4 & 0.16 \\
6FDA-durene-4BDAF (2:8) & 2.22 & 28.7 & 0.68 & 0.71 & 17.3 & 0.19 \\
6FDA-4BDAF & 1.80 & 23.3 & 0.55 & 0.68 & 11.7 & 0.21 \\
\hline
\end{tabular}

\section{S3. REFERENCE}

1. Tao, S. J. J. Chem. Phys. 1972, 56, (11), 5499-5510.

2. Lue, S. J.; Lee, D.-T.; Chen, J.-Y.; Chiu, C.-H.; Hu, C.-C.; Jean, Y. C.; Lai, J.-Y. J. Membr. Sci. 2008, 325, (2), 831-839.

3. Shantarovich, V. P.; Kevdina, I. B.; Yampolskii, Y. P.; Alentiev, A. Y. Macromolecules 2000, 33, (20), 7453-7466.

4. Williams, M. L.; Landel, R. F.; Ferry, J. D. J. Am. Chem. Soc. 1955, 77, (14), 37013707.

5. Geise, G. M.; Doherty, C. M.; Hill, A. J.; Freeman, B. D.; Paul, D. R. J. Membr. Sci. 2014, 453, (0), 425-434.

6. Thran, A.; Kroll, G.; Faupel, F. J. Polym. Sci. B Polym. Phys. 1999, 37, (23), 33443358.

7. $\quad$ Cohen, M. H.; Turnbull, D. J. Chem. Phys. 1959, 31, (5), 1164-1169.

8. Xie, W.; Ju, H.; Geise, G. M.; Freeman, B. D.; Mardel, J. I.; Hill, A. J.; McGrath, J. E. Macromolecules 2011, 44, (11), 4428-4438. 